\title{
Full and partial hydrolysis of poly(2-oxazoline)s and subsequent post-polymerization modification of the resulting polyethylenimine (co)polymers
}

Maarten A. Mees ${ }^{\mathrm{a}, \mathrm{b}}$ and Richard Hoogenboom ${ }^{\mathrm{a}, *}$

Abstract: The synthesis of linear polyethylenimine (L-PEI) is mostly performed via the acidic or basic hydrolysis of poly(2-alkyl-2-oxazoline)s (PAOx). Besides full hydrolysis leading to L-PEI, the partial hydrolysis of the PAOx sidechains results in PAOx-PEI copolymers having secondary amine groups in the polymer backbone. The secondary amine groups of L-PEI and PAOx-PEI can act as charge carrier for the complexation of DNA for gene therapy. Furthermore, they are also excellent chemical moieties for post-polymerization modification reactions providing straightforward acces to new PAOx (co)polymers based on a variety of PAOx, including the commercially available poly(2-ethyl-2oxazoline) (PEtOx). Within this review, we will discuss the acidic and basic (partial) hydrolysis of PAOx as well as the corresponding mechanisms. In addition, an overview is provided of the recent literature on the post-polymerization modification of the fully hydrolyzed L-PEI and the partially hydrolyzed PAOx-PEI.

Keywords: Linear polyethylene imine, hydrolyis, Poly(2-alkyl-2-oxazoline)s, partial hydrolysis

\section{Introduction}

Polyethylenimine (PEI) (co)polymers and its poly(2-alkyl/aryl-2-oxazoline) (PAOx) precursors have been coexisting in literature for more than 50 years, providing high potential as platform for preparing functional materials by post-polymer modification. The class of PAOx was first reported in 1966 by four independent research groups. ${ }^{1-4}$ Nowadays, the application potential of these polymers is mostly evaluated in the biomedical field as the polymers show excellent behavior in a biomedical context. ${ }^{5}$ Importantly, when PAOx interacts with a living organism no adverse reactions are observed. ${ }^{6}$ Although specific protein adsorption has been found, these proteins actually ensure that macrophages do not eliminate the polymer construct. ${ }^{7}$ These beneficial biological properties in combination with their large chemical versatility could potentially make PAOx "the new kid on the block" in the field of polymer therapeutics. The future is prosper as the first PAOx drug conjugate, SER-214, has successfully finished phase IA clinical trials. SER-214 is a PAOx-Parkinson drug developed by Serina Therapeutics allowing a once a week administration by subcutaneous injection. ${ }^{8,9}$ Many more exciting biomedical applications based on PAOx are being developed, including micellar drug delivery, ${ }^{10-13}$ polymer therapeutics, ${ }^{14,15}$ antifouling coatings ${ }^{16-18}$ and antimicrobial materials. ${ }^{19-}$ 21

Next to its high potential for biomedical applications, PAOx is also known as the most utilized precursor for preparing linear polyethylenimine (L-PEI) (IUPAC name: poly(imino ethylene)). ${ }^{22}$ L-PEI is obtained either via an acidic or basic hydrolysis of PAOx, to remove all 
the amide side chains of the PAOx. ${ }^{23}$ If only a part of the side chains is removed by partial hydrolysis of PAOx, a PAOx-PEI copolymer is obtained that contains the original polymer tertiary amide units together with the newly formed secondary amine L-PEI units (Scheme 1). ${ }^{24-26}$

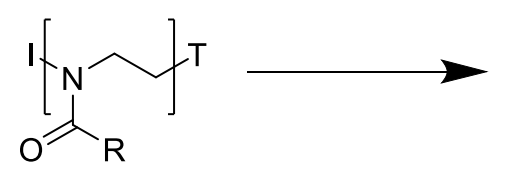

PAOx

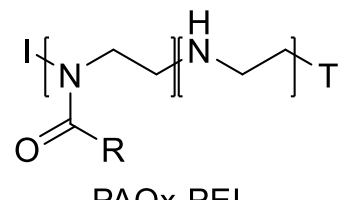

PAOx-PEI

Scheme 1. Schematic representation of the partial hydrolysis of PAOx into a PAOx-PEI copolymer containing PAOx units and the PEI units.

These secondary amine containing L-PEI and PAOx-PEI (co)polymers are cationically charged in aqueous solution making them popular for use as gene transfection vectors. ${ }^{27-30}$ Furthermore, these secondary amine units are attractive reactive handles for post-polymerization modification reactions providing an interesting route towards functional PAOx and PAOx copolymers by reaction with (activated) acids or with other comonomer structures when reacted with other nucleophiles providing a straightforward direct access to functional PAOx without the need of preparing new functional 2-oxazoline monomers.

.31-33 A well-defined PAOx homopolymer, such as poly(2-ethyl-2-oxazoline) (PEtOx) or poly(2-methyl-2-oxazoline) (PMeOx), can be first prepared or purchased. The synthesis of these polymers can be performed in a controlled way, in large scale and some of these polymers are commercially available in different grades.

Inspired by the recent interest and progress in this area, this review will first provide an overview of the synthesis of L-PEI by acidic and basic hydrolysis of PAOx. Subsequently the partial hydrolysis of PAOx yielding PAOx-PEI copolymers is discussed. The final part of the review will discuss the different post-polymerization modification reactions that have been reported using L-PEI as well as PAOx-PEI copolymers as reactive functional polymer platform to obtain functional (co)polymers.

\section{Synthesis of (L-)PEI}

PAOx and L-PEI were discovered simultaneously in $1966 .{ }^{1,3,4,34}$ Kagiya et al discovered that the hydrolysis of PMeOx yielded L-PEI by releasing the side chain as acetic acid. In a next step the L-PEI was re-acetylated with acetic anhydride demonstrating the post-polymerization modification of L-PEI, in this case yielding the original PMeOx. ${ }^{3}$ Tomalia et al reported that during the acidic hydrolysis of PMeOx in sulfuric acid an acetic acid smell was observed. Even though no full hydrolysis was obtained, it is the first report of the hydrolysis of PAOx into PAOx-PEI copolymers. ${ }^{1}$ 
In the following part the alternative methods to obtain (L-)PEI based on ring-opening of aziridines are briefly discussed (section 2.1) followed by a more thorough discussion of the full and partial hydrolysis of PAOx (sections 2.2 and 2.3).

\subsection{Ring opening polymerization of aziridines}

Long before the first L-PEI was prepared by full hydrolysis of PAOx, the synthesis of branched polyethylenimine (b-PEI) was established via the cationic ring opening polymerization of ethylenimine, also known as aziridine (Scheme 1). ${ }^{35}$ Therefore in the nomenclature, PEI generally refers to b-PEI making an explicit distinction between both the linear and branched polymers. The b-PEI was first synthesized by cationic polymerization in 1938 by I.G. Farbenindustry (Scheme 2). The b-PEI resulting from cationic ring-opening polymerization of unsubstituted aziridine (ethylenimine) contains primary, secondary and tertiary amino groups in a 1:2:1 ratio resulting from ring-opening polymerization of ethylenimine as well as chain transfer to polymer and termination reactions. ${ }^{36}$ In contrast, the L-PEI only contains secondary amino groups. The b-PEI is water soluble and is $20 \%$ protonated at physiologic $\mathrm{pH}$, depending on the polymer concentration.

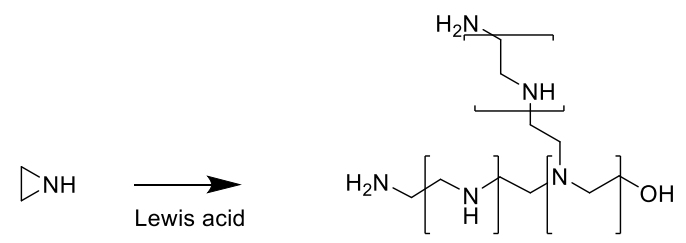

Scheme 2. Schematic representation of the ring opening polymerization of aziridine resulting in b-PEI.

The cationic ring-opening polymerization of $N$-substituted aziridines followed by side chain hydrolysis is another option to obtain linear PEI (Scheme 3). ${ }^{37}$ The aziridines can be substituted with different bulky groups lowering the reactivity for the chain transfer reactions, which promotes the formation of a linear polymer even though $100 \%$ linearity can hardly be reached. Aziridines substituted with different functionalities, such as alkyl, benzyl, sulfonyl, and perfluoro acyl groups have been polymerized. In a final step, some of these linear polyaziridines could be deprotected via cleavage of the side chain yielding linear PEI. ${ }^{38-40}$ The branching was minimized by Goethals et al. using $N$-(2-tetrahydropyranyl)aziridine (THPA), which could be cleaved under mild conditions to obtain L-PEI. ${ }^{41,42}$ The polymerization of activated sulfonamide aziridines via living anionic polymerization was recently explored by Bergman $^{43}$ and Wurm et al. ${ }^{42,44,4546}$ The obtained polyaziridines or polysulfonamide could partially (desulfonylated) to obtain L-PEI either via hydrolytic cleavage ${ }^{47}$ or via reductive removal with a catalyst. ${ }^{42}$ 


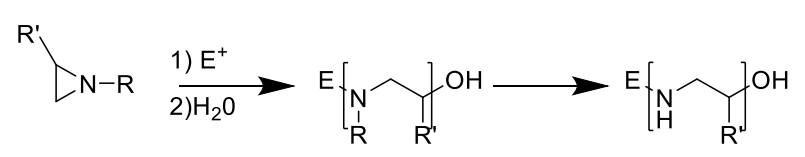

Scheme 3. Ring opening polymerization of substituted aziridine and subsequent deprotection obtaining L-PEI.

\subsection{The hydrolysis of PAOx}

To date, the best method to obtain L-PEI is the full hydrolysis of PAOx under either basic or acidic conditions. L-PEI can exist in two different types depending on the protonation state. The protonated L-PEI is water soluble while the free base L-PEI is only soluble in water when the temperature exceeds the melting point of the L-PEI $\left(50-68^{\circ} \mathrm{C}\right) .{ }^{48}$ As the free base form is insoluble in water at room and body temperature and the protonated form is water soluble at those temperatures, the $\mathrm{pH}$ of the solution strongly influences the solubility and applicability of L-PEI. However, there is no straightforward description of the pKa value of L-PEI as the large amount of amine groups heavily influence each other leading to a broad pKa range. ${ }^{49,50}$ In practice, when an acidic solution of L-PEI is basified by titration with a $\mathrm{NaOH}$ solution, the polymer solution becomes turbid accompanied by a drop in $\mathrm{pH}$, at $\mathrm{pH} 9.4$ indicating the crystallization of L-PEI, as reported by Goethals and coworkers. ${ }^{51}$

The preparation of L-PEI by hydrolysis of PAOx generally starts with PMeOx or PEtOx as parent polymer due to the commercial availability of the monomers and polymer (PEtOx) in large quantities, atom economy and good water solubility. The first reported reaction conditions for the hydrolysis of PAOx were often under alkaline $(\mathrm{NaOH})$ conditions at $98^{\circ} \mathrm{C}$ for $3 \mathrm{~h} . .^{22,52}$ In addition to the above mentioned polymers, both poly(2-phenyl-2-oxazoline) $\mathrm{PPhOx}$ and poly(2-isopropyl-2-oxazoline) ( $\mathrm{PiPrOx}$ ) have been used for the acidic hydrolysis into LPEI. ${ }^{53,54}$ In the following part, the basic and acidic hydrolysis of PAOx are discussed in further detail.

\subsubsection{Basic hydrolysis}

The basic hydrolysis of PAOx was the first reported method for the preparation of L-PEI (Scheme 4). ${ }^{3,55,56}$ The reaction follows second order kinetics (rate $=k 2\left[\right.$ amide] $\left[\mathrm{OH}^{-}\right]$). In the first step the $\mathrm{OH}^{-}$attacks the carbonyl function and subsequently this tetrahedral intermediate is deprotonated and the carboxylate is irreversibly released.

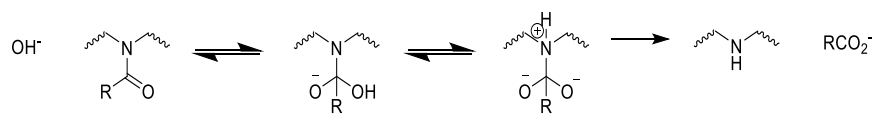

Scheme 4. Basic hydrolysis of PAOx: The basic hydrolysis of the PAOx starts with the attack of the $\mathrm{OH}^{-}$on the amide bond forming the tetrahedral intermediate. After protonation of the nitrogen there is an irreversible release of the carboxylate. 
Tanaka et al. reported an attempt for the basic hydrolysis of PPhOx that, however, failed. ${ }^{53}$ The basic hydrolysis of PMeOx and PEtOx can be controlled by the amount of base added as was shown by Chujo et al. ${ }^{57}$ Not only PEtOx and PMeOx can be used for the synthesis of L-PEI, but also poly(2-H-2-oxazoline) PHOx, ${ }^{58,59}$ where the $\mathrm{R}$ group on the side chain is a hydrogen atom, can be hydrolyzed to L-PEI under basic conditions. ${ }^{60}$

A major drawback of this basic hydrolysis approach is, however, that partial backbone degradation occurs during hydrolysis. ${ }^{61}$ This backbone degradation has long been overlooked as the resulting L-PEI could not be analyzed by size exclusion chromatography (SEC) as it tends to strongly interact with the column materials. Only with hexafluoroisopropanol as eluent it was found to be possible to perform SEC revealing the presence of significant low molar mass shoulders in the SEC traces of the obtained L-PEI after basic hydrolysis of PAOx.

\subsubsection{Acidic hydrolysis}

The acidic hydrolysis is more promising than the basic hydrolysis as the protonated (partially) hydrolyzed copolymer remain soluble. The first acidic hydrolysis of PEtOx, PMeOx and $\mathrm{PPhOx}$ was shown in 1979 by Kem et al using aqueous $\mathrm{HCl}$ (Scheme 5). ${ }^{22}$ As the hydrolysis is mostly done in large excess of $\mathrm{HCl}$ it follows pseudo-first order kinetics. During the acid hydrolysis the amides are activated by the protonation of the carbonyl functionality and the formed amines are protonated due to the high acid concentration, ${ }^{51,62}$ which heavily influences the reaction speed and the cleavage of the side chain. The protonation of the PEI units plays an important role because this influences the hydrolysis and solubility in water.

The acidic hydrolysis of $\mathrm{PMeOx}^{22}$ and PEtOx ${ }^{62,63}$ was investigated in a more detailed way. In a first step (Scheme 5), the amide is protonated making the attack of water easier. The nucleophilic attack of the water is the rate determining step. The formed tetrahedral intermediate is promptly converted to the amine under the release of a carboxylic acid. This is accompanied by the corresponding smell of either acetic acid from PMeOx, propionic acid from PEtOx or in the case of poly(2-n-propyl-2-oxazoline) (PPrOx), butyric acid. ${ }^{25}$ As discussed, the formed PEI is protonated due to the acidic conditions (pKa 10), although it is difficult to protonate all the amine groups due to repulsion effects when the hydrolysis is pushed to full conversion. Nonethless, the acidic hydrolysis of PAOx is an accurate and reproducible method to obtain L-PEI. The temperature, however, plays a crucial role as the hydrolysis is accelerated when the temperature rises. It was however also shown that at higher temperatures, above $180^{\circ} \mathrm{C}$ under pressurized conditions, the polymer backbone degrades during acidic hydrolysis. ${ }^{62}$ Due to the protonation of the formed amines after hydrolysis, there is an effective consumption of protons implying that the overall $\mathrm{pH}$ will increase and when using less acidic conditions the amount of acid can be used to control the hydrolysis conversion. It may be anticipated that there will be the possibility to form new L-PEI units even when all 
acid is used to protonate the formed L-PEI units, but this will be slow due to the slow exchange of the protons. In normal time frames this will not happen (hours). ${ }^{62}$
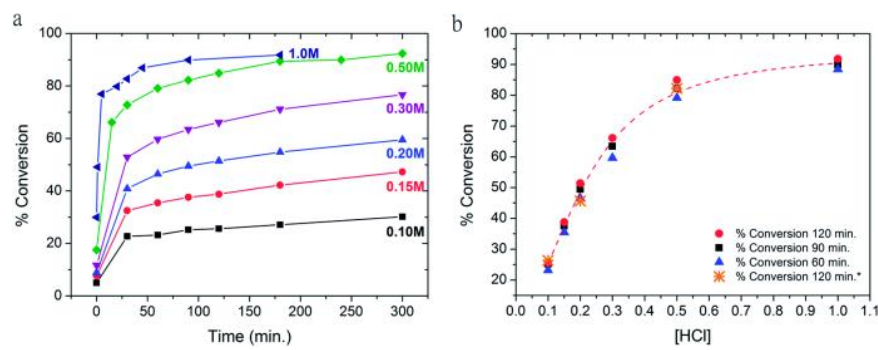

Figure 1. (a) Conversion versus time plot for the hydrolysis of PEtOx $3 \mathrm{kDa}$ (amide concentration $1 / 4$ [A] $1 / 40.48 \mathrm{M}$ ) at $180^{\circ} \mathrm{C}$ under various $\mathrm{HCl}$ concentrations ranging from $0.10 \mathrm{M}$ to $1.0 \mathrm{M}$. The degree of hydrolysis levels off at defined values at each acid concentration, therefore allowing good control over the degree of hydrolysis. (b) Conversion plotted against $\mathrm{HCl}$ concentration. * Reactions performed in closed pressure tubes and conventional heating instead of microwave heating. The robustness of the partial hydrolysis is demonstrated by the small impact that variations of 30 minutes of reaction time have on the degree of hydrolysis. Reproduced from de la Rosa VR, Bauwens E, Monnery BD, De Geest BG, Hoogenboom R. Fast and accurate partial hydrolysis of poly(2-ethyl-2oxazoline) into tailored linear polyethylenimine copolymers. Polym Chem [Internet]. 2014 [cited 2014 Jun 17];5:4957-64. Available from: http://xlink.rsc.org/?DOI=c4py00355a with permission of The Royal Society of Chemistry. ${ }^{62}$

Next to this discussed hydrolysis mechanism, the acidic hydrolysis of amide side chains may be accelerated by the formation of seven ring like intermediates with neighbouring L-PEI groups. The proton on the protonated amine intramolecularly hydrogen bonds with the amide making it more susceptible to a nucleophilic attack. This is evidenced by the acceleration of the reaction at the beginning of the hydrolysis of PEtOx ${ }^{63}$ and PPrOx after the formation of the first L-PEI units. ${ }^{25}$ The hydrolysis of PEtOx with different chain lengths showed that the degree of polymerization (DP) has no significant influence on the rate of hydrolysis and on the molar mass distribution (i.e dispersity) of the resulting polymer. In the same study it was also shown 
that the PMeOx hydrolyzes faster than PEtOx, whereby the kinetics showed that PMeOx had linear kinetics until $80 \%$ and PEtOx up to $60 \%$ due to the better solubility of the first.
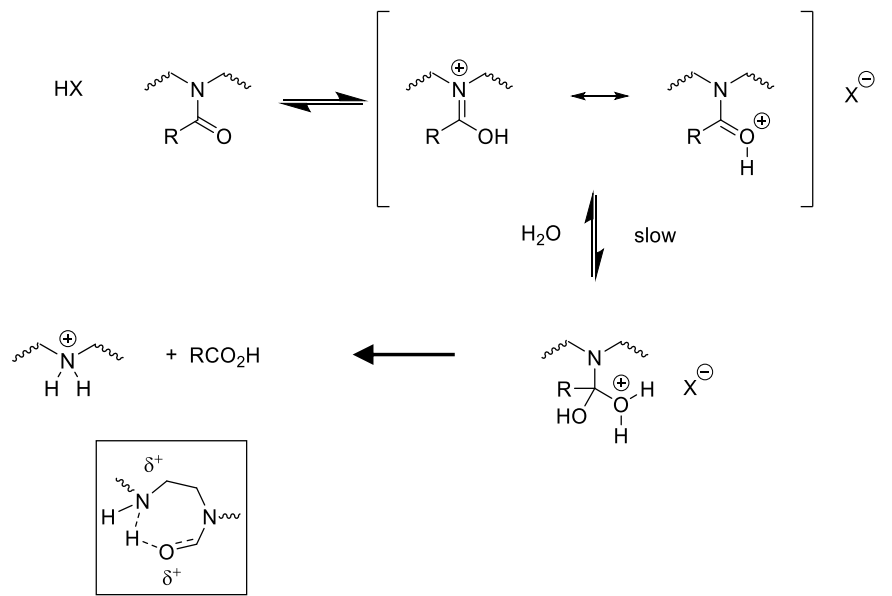

Scheme 5. Acid hydrolysis of PAOx: The acid hydrolysis starts with a protonation followed by the formation of the tetrahedral intermediate via water. This step is the rate determining step. The last step removes the carboxylic acid from the polymer obtaining the PEI. In the square at the bottom the seven ring is shown which may facilitate the hydrolysis of an amide that is next to a L-PEI unit.

The polymer concentration was found to not affect the hydrolysis rate, at least under very acidic conditions where pseudo first order kinetics are achieved. ${ }^{64}$ The acid concentration does play a role as protons are consumed during the hydrolysis as mentioned before. ${ }^{65}$ As a result, the amount of acid can be used to control the amount of PEI units formed in partially hydrolyzed copolymers, ${ }^{62,66}$ providing a controlled and robust way to make partially hydrolyzed polymers with a certain amount of PEI as will be further discussed in the following section.

\subsubsection{Partial hydrolysis of PAOx}

The above discussed hydrolysis reactions mainly focused on full hydrolysis of PAOx to obtain L-PEI. However, the hydrolysis can also be stopped at incomplete conversion to obtain copolymers with a certain degree of PEI units together with remaining PAOx units yielding PAOx-PEI copolymers (Figure 1). ${ }^{62}$ The partial hydrolysis of PAOx was first demonstrated by Kem in 1979 and they already hypothesized that the new polymers could be used for copolymer synthesis. ${ }^{22}$ More recently, the obtained PEtOx-PEI copolymers have been demonstrated to be still biocompatible up to $25 \%$ hydrolysis. ${ }^{63}$

Different methods have been explored for the synthesis of block PEI-PAOx copolymers. The difference in solubility of different PAOx has been explored to induce selective hydrolysis of PAOx block copolymers by combining a hydrophilic block, PMeOx or PEtOx, with a more hydrophobic block. Both triblock and diblock copolymers have been used in this approach. ${ }^{23,61,67}$ The selective partial hydrolysis of triblock or diblock copolymers having poly(2-(4-t-butylphenyl)-2-oxazoline) or poly(2-undecyl-2-oxazoline) as hydrophobic block(s) and PEtOx as hydrophilic (middle) block showed promising results. ${ }^{65}$ Via this method, 
block-like L-PEI parts could be synthesized due to preferential complete hydrolysis of the hydrophilic PEtOx part while only around $25 \%$ of the hydrophobic parts were hydrolyzed. To obtain a more selective hydrolysis, a lower PEtOx concentration and lower temperatures were used. ${ }^{65}$ As mentioned in the basic hydrolysis part, PHOx polymers can also be hydrolysed into L-PEI. As the unsubstituted side chain of this polymer is less bulky, its hydrolysis is faster compared to $\mathrm{PMeOx}$ and PEtOx. As a result, the hydrolysis of copolymers consisting of PHOx and PEtOx in $\mathrm{NaOH}$ enabled selective hydrolysis of the PHOx resulting in PEtOx-PEI copolymers. ${ }^{68}$

Some efforts have been made to perform acidic hydrolysis in solvent mixtures with water. A study was reported on the acidic hydrolysis of PMeOx, PEtOx, PPhOx in 80-20 (w-w\%) ethanol water mixtures. The experiments with PMeOx-b-PPhOx and PMeOx-b-PEtOx block copolymers showed that the $\mathrm{PMeOx}$ side chain was preferentially cleaved up to full conversion while the PPhOx was only partially removed up to $10 \% .^{23,65,67,69}$ The hydrolysis of a PMeOxb-PEtOx block copolymer was less selective, although the PMeOx was still hydrolyzed faster than the PEtOx block. The polymers containing L-PEI can subsequently be used in postpolymerization modification reactions.

\section{Post-polymerization modification of L-PEI}

Post-polymerization modifications, also referred to as polymer analogous reactions, of L-PEI are straightforward as the secondary amine groups act as a nucleophile providing straightforward access to range of (co)polymers via, e.g. coupling of carboxylic acids, nucleophilic substitution, reductive alkylation/amination, ring opening and Michael addition reactions. In the following, the reported post-polymerization modification reactions for L-PEI will be discussed. The modification of L-PEI is mostly performed on part of the secondary amino groups to get partially functionalized L-PEI that combined the polyelectrolyte properties of PEI with the properties of the newly added side chains. The following part does not aim to be a comprehensive overview of all reported modification reactions, but rather provides illustrative examples of the most common modification routes.

\subsection{Post-polymerization modification with carboxylic acids}

The coupling of the secondary amino groups with a carboxylic acid can be performed with a wide variety of coupling agents that are commonly used, e.g. in peptide synthesis, to increase the reactivity of the carboxylic acid (scheme 6). 


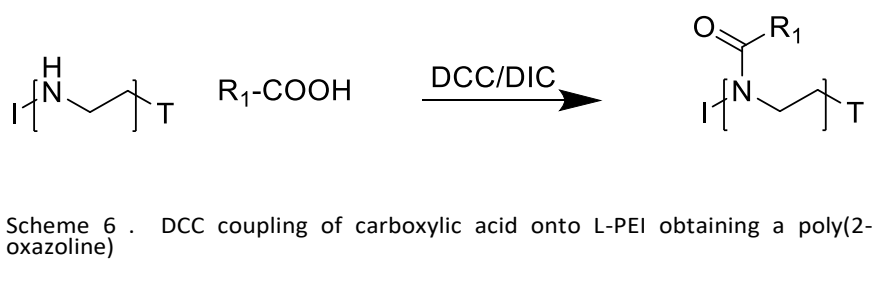

The synthesis of PEI-g-poly(ethylene glycol) (PEG) graft copolymers was reported using (1ethyl-3-(3-dimethylaminopropyl)carbodiimide) (EDC) as coupling agent to attach acidfunctionalized PEG to the the secondary amine groups of L-PEI, obtaining a $10 \%$ coupling rate. The obtained polymers were used in nasal delivery. ${ }^{70}$ Dextran modified with a carboxylic acid was also coupled to the amine of L-PEI via EDC coupling showing better gene transfection than the dextran coupled via reductive amination with degrees of substitution (DS) ranging from 0.06-0.18. ${ }^{71}$ The preparation of L-PEI containing nucleic acids has been reported using ( $N, N^{\prime}$-dicyclohexylcarbodiimide) (DCC) as coupling agent for the reaction with the 2carboxyethyl derivate of the nucleic acid. ${ }^{72}$

Besides the coupling of an acid to the secondary amino groups in presence of a coupling agent, pre-activated carboxylic acids can also be coupled to L-PEI. For example, $N$-succinimidyl-4pentenoate has been coupled to L-PEI to introduce a double bond in parts of the side chain $(27 \%)$. This double bond was used in a thiol-ene photo addition reaction to couple Lglutathione yielding L-PEI partially modified with L-glutathione, which was demonstrated to efficiently cross of the blood brain barrier. ${ }^{73}$ Hydrogels were made for the capturing of DNA using a similar L-PEI modification strategy. The amine on the L-PEI was modified with succinimidyl-4-pentenoate yielding a polymer with a double bond in the side-chain. The double bond was then used to make hydrogels via radical thiol-ene chemistry and a diol cross-linker. ${ }^{74}$ L-PEI has also been grafted with nucleic acids, whereby the first attempt based on coupling a propionic acid derivate of the nucleic acid with DCC onto L-PEI failed. However, when a $p$ nitrophenyl activated ester derivative was first prepared and used for the coupling reaction, the reaction speed was increased due to the nitrophenyl ester being a better leaving group leading to successful coupling. A similar coupling reaction was successful performed with nucleic acids containing an acid chloride or bromide containing adenine or thymine as activated ester. $^{75-78}$

\subsection{Post-polymerization modification by nucleophilic substitution reactions}

During the nucleophilic substitution of L-PEI, the free electron pair of the secondary amine is used for the nucleophilic attack on an electrophile, such as the activated carboxylic acids that were discussed in the previous section.

The most straightforward other nucleophilic substitution of L-PEI is the alkylation with an alkyl halide in the presence of a base. (Scheme 7) 


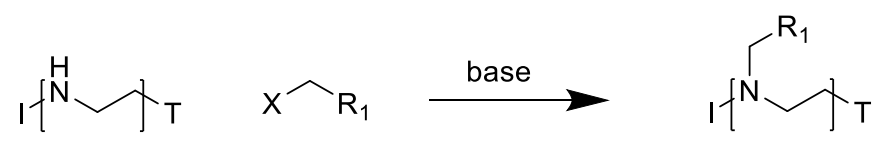

Scheme 7. Nucleophilic substitution of an alkyl halide obtaining a tertiary amine

However, this strategy involves the possibility of partial overalkylation leading to the formation of quaternary amine groups, especially when using small alkyl halides, such as methyl iodide. ${ }^{79}$ The preparation of benzylated L-PEI via the nucleophilic substitution with the bulkier benzyl bromide has been reported as efficient alkylation method. ${ }^{79}$ For the synthesis of chelating polymers a styrene-graft-PEI copolymer was carboxylated by coupling of chloroacetic acid to the PEI units. ${ }^{80}$ The reaction between a bromide mesogenic reagent and subsequent quaternization has been reported to lead to the formation of liquid crystalline polymers. ${ }^{81} \mathrm{~A}$ similar method was reported to alkylate L-PEI via nucleophilic substitution with 4(5)-bromo methylimidazole. $^{82}$ Alternatively, 4(5)-(hydroxymethyl)-imidazole has been used as electrophile in the presence of a base for the preparation of imidazole functionalized L-PEI. These imidazole-functionalized L-PEIs were shown to act as ribonuclease A mimicking RNA. ${ }^{83} /$

Another nucleophilic modification reaction of PEI was indirectly shown in industrial gas scrubbers that remove $\mathrm{CO}_{2}$ from gas waste. The amines of PEI are prone to react with $\mathrm{CO}_{2}$ to reversibly form carbamates. It was however shown that these carbamates groups form cyclic ureas with neighbouring secondary amino groups making the regeneration impossible. ${ }^{84}$ This side reaction is enhanced by increasing the gas stream moist. ${ }^{85}$

\subsection{Post-polymerization modification by ring opening reactions}

A special case of nucleophilic substitution can be found with the ring opening of electrophilic reagents, such as epoxides, Traut's reagent and cyclic sulfones. (Scheme 8)
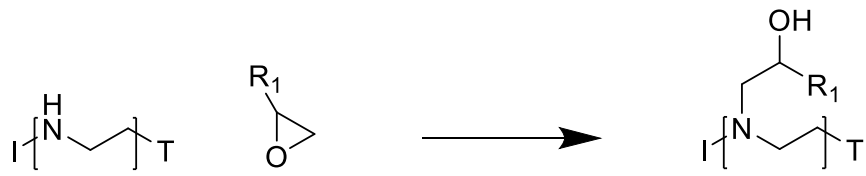

Scheme 8. Ring opening of an epoxide with L-PEI yielding the corresponding tertiary amine.

Alkane modified L-PEI copolymers were prepared by reacting part of the secondary amine groups with 1,2 epoxydecane. ${ }^{86}$ The reaction of PEI with ethylene oxide yielded a copolymer having tertiary amines in the backbone and an alcohol in the side chain. ${ }^{40}$

The synthesis of sulfhydryl functionalized L-PEI has been reported by ring-opening reaction of L-PEI with Trauts reagent (2-iminothiolane). ${ }^{87}$ In this report, L-PEI-dexamethasone was subsequently formed by reacting the thiol containing L-PEI with dexamethasone-21-mesylate and the resulting copolymer was evaluated as gene delivery vector. Mercapto-ethylation of L- 
PEI has been achieved by reaction with ethylene sulfide and carbon disulphide. ${ }^{88} \mathrm{~L}-\mathrm{PEI}$ is also promising for use in polymer electronics and was therefore grafted with lithium conductors by ring-opening reaction of 1,3-propane sulfone. ${ }^{89}$

\subsection{Post-polymerization modification by reductive amination}

In a reductive amination, the amine is used for nucleophilic attack on a ketone or aldehyde yielding an imine that is subsequently reduced in situ, yielding a stable tertiary amine. One of the first reports on the post-polymerization modification of PEI was reported using the Eschweiler-Clarcke $N$-methylation, whereby the reaction between the amino group and formaldehyde in presence of formic acid as reducing agent yields the methylated PEI (Scheme 9). ${ }^{53,90}$ More recently Lambermont-Thijs et al described the preparation of methylated L-PEI synthesized via this reductive amination method. ${ }^{79}$ A major advantage of this reductive amination method over alkylation with e.g. methyliodide is that no overalkylation occurs, as the formed tertiary amino groups are no longer reactive towards aldehydes. The reaction of PEI with less than 0.5 equivalents of paraformaldehyde polymers has been reported to yield hydroxymethylamine side chains while larger amounts of formaldehyde lead to the formation of alkylated PEI (Scheme 6).

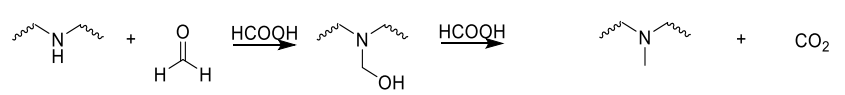

Scheme 9. Introduction of formaldehyde on PEI yielding hydroxymethylamino modified L-PEI when using less than 0.5 equivalents of formaldehyde and further reductive amination in larger excess of formaldehyde in presence of formic acid as reducing agent.

The polysaccharide dextran was also coupled to PEI via reductive amination of the aldehyde groups onto the amines of PEI. Even though the synthesis was successful, the related polymers prepared via EDC coupling of dextran onto PEI showed more efficient gene transfection, however a higher DS-range was obtained (DS 0.13-0.38). ${ }^{71}$

\subsection{Post-polymerization modification by oxidation}

The amines on the L-PEI backbone can also be modified via an oxidation step. ${ }^{33,91}$ Different oxidizing groups were evaluated revealing that the use of hydrogen peroxide efficiently yields hydroxylamine groups with a degree of oxidation ranging from $20-85 \%$ depending on the concentration. Interestingly, these hydroxylamino groups are proposed to isomerize via nitrone and oxaziridine intermediates to the most stable amide form. Importantly, the formed amide group is located in the backbone of the L-PEI, enabling backbone degradation of the copolymers. ${ }^{33}$ 


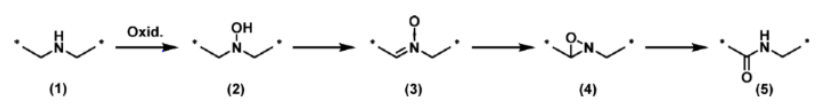

Scheme 10. Schematic Representation of the Oxidation Mechanism of a Secondary Amine (1) via Hydroxylamine (2), Nitrone (3), and Oxaziridine (4) Intermediates To Form the Final Amide Group (5) Reprinted with permission from (Englert, C. et al. Enhancing the Biocompatibility and Biodegradability of Linear Poly(ethylene imine) through Controlled Oxidation. Macromolecules 48, 7420-7427 (2015).). Copyright (2018) American Chemical Society. ${ }^{33}$

\subsection{Post-polymerization modification by Michael addition}

During the Michael addition reaction, amines are coupled to $\alpha-\beta$ unsaturated carbonyl compounds. PEI-grafted with a methyl ester containing side chain was prepared by Michael addition of methyl methacrylate onto L-PEI, the DS can be tuned between 0 and 1 and is limited by the addition of the reactant. ${ }^{92,93}$ The methacrylate reacted with the amine groups and subsequently, the ester groups could be either amidated or hydrolyzed. In a similar approach the polymer could change its charge by hydrolyzing the ester towards the carboxylic acid yielding a zwitterionic polymer. Polystyrene-graft-PEI was used for the Michael addition reaction with acrylic acid, directly yielding a zwitterionic copolymer structure, the DS ranged between 47.4 and $100 \% .{ }^{80}$ The coupling of a histidinylated L-PEI was achieved by coupling a methacrylate modified histidine, by varying the $N$-acryloyl amino acids different ranges of substitution were obtained starting from $7.4 \%$ up to $67 \% .{ }^{94}$

\section{Post-polymerization modification of poly(2-oxazoline)-poly(ethylene imine) copolymers}

The hydrolysis of PAOx can be stopped before complete conversion is reached yielding PAOxPEI copolymers that contain both tertiary amide groups from PAOx and secondary amino groups from L-PEI. These amino groups have similar reactivity as discussed above for L-PEI and can be used in a post-polymerization modification reaction, which reveals several advantages compared to the copolymerization route for preparing functional PAOx. First, new monomers have to be synthesized for copolymerization, which is a large burden from both synthetic and purification point of view. The introduction of new monomers makes it more challenging to obtain well-defined (co)polymers with narrow dispersity as even minor impurities can interfere with the CROP. Some functional groups, especially the nucleophilic groups, need to be protected in the monomer as otherwise they would terminate the CROP. Finally, the reactivity of new monomers can vary, leading to composition drift throughout the polymerization resulting in the formation of gradient copolymers which can, however, also be advantageous in some cases. ${ }^{95}$ In favor of the copolymerization approach is the good control over the amount of functional monomers based on stoichiometry of the comonomers as well as 
the fact that no other functionalities are present due to e.g. partial functionalization that may occur during incomplete post-polymerization modification.

The polymerization of EtOx, MeOx and PrOx is highly optimized and these polymers are easily accessible in larger quantities, with high MW and/or low $Đ$, making them appealing as precursors for PAOx-PEI and further post-polymerization modification. ${ }^{96}$ By submitting such PAOx homopolymers to a partial hydrolysis, a controlled amount of secondary amino groups can be introduced, albeit this is mostly kinetically controlled. A more robust control over degree of hydrolysis was reported based on variation of the amount of acid or base during hydrolysis. 62

Figure 2 provides an overview of the most common post-polymerization modification reactions that were reported for PAOx-PEI copolymers. The amines on the PEI unit function as a nucleophile. Starting from the right and anticlockwise, the partial hydrolysis of PAOx towards PAOx-PEI copolymers is shown, followed by modification with acid chlorides, reductive amination of aldehydes, DCC/ $N, N$-diisopropylcarbodiimide (DIC) coupling of carboxylic acids, urea formation via (iso)cyanate coupling and ring opening of epoxides. In the following part, illustrative examples of such modification reactions will be discussed, whereby we do not aim to provide a comprehensive overview of all literature reports, but would rather like to give a flavor of the wide range of possibilities to inspire further future modification reactions.

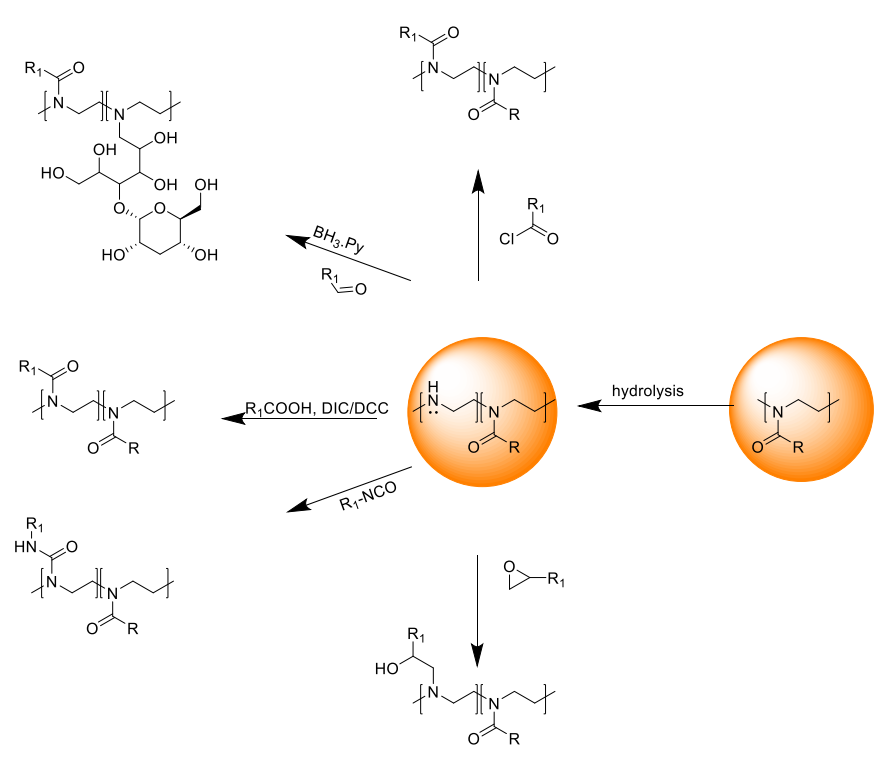

Figure 2. Overview of illustrative examples of the post-polymerization modification reactions of PAOx-PEI that were reported. Starting from the right and anticlock wise, the partial hydrolysis of PAOx, an acid chloride reaction, reductive amination of an aldehyde, DCC/DIC coupling of a carboxylic acid, urea formation via (iso)cyanates, ring opening of epoxides

\subsection{Post-polymerization modification with carboxylic acids}

Similar to the modification of L-PEI homopolymers, PAOx-PEI copolymers can be modified by reaction with activated carboxylic acids. Carbodiimides such as DIC and DCC can be used for in situ activation of the carboxylic acids. PMeOx-hydrogels were developed by partial 
hydrolysis of PMeOx followed by the DCC coupling of a carboxylic acid functionalized anthracene that contained a disulfide in the linker. These polymers were used to form two types of hydrogels, namely photosensitive hydrogels based on photodimerization of the anthracene moieties (Figure 3) and redox responsive hydrogels having disulfide crosslinks for which the anthracene acted as leaving group. The amine group conversion is high as yields of conversion of 77 up to $100 \%$ are obtained ${ }^{97}$ Next to this anthracene-based disulfide linker, hydrogels were prepared with different disulfide linkers that were coupled to PMeOx-PEI via the carboxylic acid groups of 3,3'dithiodipropanoic acid, the acid chloride of 3,3'dithiodipropanoic acid and a disulfide linker with isocyanates. Due to the presence of the disulfide bridge, reductive conditions such as sodium borohydride or triphenylphosphine were used to degrade the hydrogels into the linear polymer building blocks. Similar polymers and reaction conditions as the photo-gelation work ${ }^{97}$ mentioned in the start of this paragraph resulted in a range between $77 \%$ and $99 \%$ amine conversion. ${ }^{98}$

The same authors introduced 2,2'-bipyrydines in the side-chains of PAOx by DCC coupling of an acid-containing 2,2'-bipyridine derivative to PAOx-PEI yielding a thermally and redox responsive hydrogel upon metal complexation with either $\mathrm{Mn}^{2+}, \mathrm{Fe}^{2+}$ or $\mathrm{Co}^{2+}$ ions. ${ }^{99,100}$ Coumarin containing hydrogels were also developed by using DCC coupling to the PEI part of PAOx-PEI copolymers. The DCC coupling indeed shows high conversion yield as showed previously, 72-99\%. ${ }^{98,99}$ The coumarin dye can photoreversibly dimerize to form a cyclobutane ring inducing gelation. ${ }^{56}$ Similar fluorescent polymers are obtained by coupling of pyrene both to $\mathrm{PMeOx}$ and to PEtOx. The pyrene contained a carboxyl functionality and was coupled to PAOx-PEI via DIC coupling. This DIC coupling has slightly lower conversion yields between $71 \%$ and $92 \% .^{56}$

In another study, furan and maleimide were introduced into PAOx-PEI by DCC coupling and, subsequently, the Diels Alder reaction between both was explored for the formation of hydrogels. ${ }^{101}$ Both components were coupled successfully in high conversion rates, although there is a slight difference between both maleimide and furan coupling as the maleimide conversion ranged from $79-92 \%$ while the furan conversion ranged from $72-80 \% .{ }^{101}$ Also a DCC coupling onto carbon nanotubes containing carboxylic acids was shown with PMeOxPEI, the NMR analysis indicate full conversion of the amines. ${ }^{102}$ Similar as with PEI homopolymers, the coupling of nucleotides to PAOx-PEI was demonstrated via a DCC coupling of thymine propionic acid. ${ }^{76}$ 


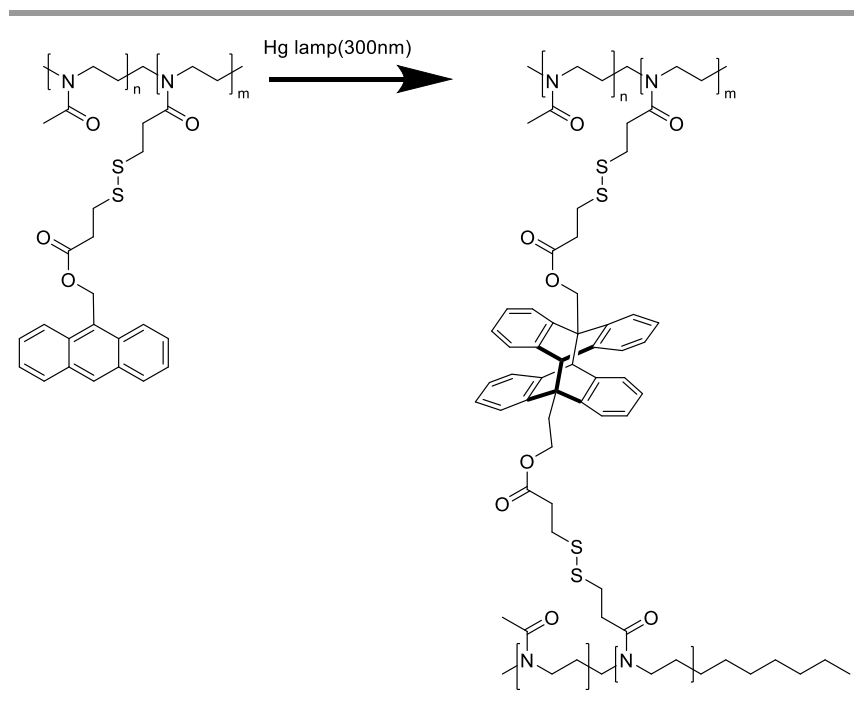

Figure 3. Post-polymerization modification of PMeOx forming an anthracene bridged system. ${ }^{97}$

The nucleophilic attack of the secondary amine of PAOx-PEI onto an acid halide can also be used to reinstall a functional poly(2-oxazoline) side chain. Methyl succinyl chloride was coupled onto a PEtOx-PEI polymer to introduce methyl ester groups into the side chains of the copolymers. This methyl esther could subsequently be transformed into various amides by direct amidation, here full conversion is obtained. ${ }^{103}$ The reaction of diacid chlorides with PAOx-PEI in presence of a base catalyst, e.g. 1,8-diazabicycloundec-7-ene (DBU), leads to the formation of crosslinked polymer networks with a $80 \%$ yield. ${ }^{98}$ Diacid chloride(adipoyl chloride) was used as a cross linker to form $\mathrm{PMeOx}$ hydrogels. The hydrogel formation was quantified by removing unreacted polymer via soxhlet extraction and showing a yield in a broad range, between $43-100 \% .^{57}$

\subsection{Post-polymerization modification by reductive amination}

As discussed for L-PEI homopolymers, the secondary amino groups can be reacted with aldehydes to form an imine linker that is in situ reduced to the tertiary amine. Carbohydrate containing polymers were obtained by reductive amination of glucose and maltose onto PEtOxPEI copolymers with different degrees of hydrolysis. The aldehyde of the ring opened carbohydrate was transformed into an imine due to the nucleophilic attack of the amine onto the aldehyde and subsequent reduction of the newly formed bond pushing the reaction to completion in high yield. ${ }^{31}$

\subsection{Post-polymerization modification with isocyanates}

The reaction of PAOx-PEI with isocyanates leads to the formation of an urea bond on the sidechain. Isocyanates were used to form redox sensitive PAOx-PEI based hydrogels by reacting a bifunctional isocyanate that contained a disulfide bond in a $80 \%$ yield ${ }^{98}$ Hydrogels containing urea bonds were synthesized by using PMeOx-PEI and hexamethylene diisocyanate as a cross linker with only $55 \%$ gel formation. ${ }^{57}$ Pyrene containing hydrogels were obtained 
via crosslinking with hexamethylene diisocyanate in the presence of DBU, the coupling of the pyrene was achieved via DCC coupling as already described in the carboxylic acid coupling part. $^{104}$

\subsection{Post-polymerization modification by ring-opening reactions}

Lecommandoux and coworkers found a method for making hydrogels starting from PEtOxPEI in combination with a bisglycidyl ether (1,6 hexanediol diglycidyl ether) with two epoxides as crosslinker. These epoxides are attacked by the amine leading to the formation of a tertiary amine having an hydroxyl group on the beta-position of the side chain. ${ }^{105} \mathrm{~A}$ similar approach was used for the formation of a degradable hydrogel and nanogel particles by using hydroxyethyl disulfide-bis-di-glycidyl ether. These nanogels showed a dual-responsive character as the addition of an acid protonated the remaining tertiary amino groups inducing swelling while the addition of a reductive agent cleaved the nanogel by breaking the disulfide bonds. ${ }^{106}$

\section{Conclusions}

The applications of biomedical polymers are expanding and these new biomedical challenges requires the incorporation of new and multiple functionalities along the polymer chain. The synthesis of PAOx by living CROP already provides a wide palette for the preparation of functional PAOx. However, side chain modification and functionalization through CROP is limited by the necessity of synthesizing novel monomers and to protect nucleophilic functional groups. These polymerizations introduce a certain control over the monomer distribution along the chain, with either gradient, block or random monomer distribution. ${ }^{107}$

The two-step-modification of PAOx homopolymers by (partial) hydrolysis and subsequent functionalization of the resulting secondary amine groups has become a popular alternative. This post-polymerization modification approach is based on well-defined homopolymers, such as PEtOx and PMeOx that are either commercially available or easy to prepare based on the commercially available monomers. The advantage of this two-step modification comes from a lower synthetic burden, straightforward chemistry and no optimization of the polymerization required while the possibility to have these polymer in large scale make them ideal for commercial applications. Despite these advantages some disadvantages appear, the necessity of the extra hydrolysis step, possible backbone degradation in basic condition, no knowledge about the PEI distribution along the chain and sensitivity of acid/basic end groups. Copolymers of more specialized polymers such as PPrOx can be modified in a similar way and this could be potentially interesting for further research

The L-PEI and PAOx-PEI platforms have been widely exploited for further modification using various coupling chemistry, including nucleophilic substitution, reductive amination, ring opening, DCC or EDC coupling and Michael addition. This makes it possible to make copolymers with nucleophilic groups which might be difficult or impossible to polymerize. 
This review has demonstrated the potential of this post-polymerization approach by discussing relevant examples, thereby aiming to serve as inspiration for future work in this area as a large number of either modification reactions can be developed and the resulting (co)polymers can be utilized for a broad range of (biomedical) applications.

\section{Conflicts of interest}

Conflict of interest: $\mathrm{RH}$ is one of the founders of Avroxa BVBA that commercializes poly(2oxazoline)s as Ultroxa ${ }^{\circledR}$. MAM has no conflicts to declare.

\section{References}

(1) Tomalia, D. a; Sheetz, D. P. J. Polym. Sci. Part A-1 Polym. Chem. 1966, 4 (9), 2253.

(2) Seeliger, W. H. H.; Aufderhaar, E.; Diepers, W.; Feinauer, R.; Nehring, R.; Their, W.; Hellmann, H. Polym. Lett. 1966, 5 (1), 871.

(3) Kagiya, T.; Maeda, T.; Fukui, K.; Narisawa, S. Polym. Lett. 1966, 4, 441.

(4) Levy, A.; Litt, M. Polym. Lett. 1967, 5, 881.

(5) Bauer, M.; Lautenschlaeger, C.; Kempe, K.; Tauhardt, L.; Schubert, U. S.; Fischer, D. Macromol. Biosci. 2012, 12 (7), 986.

(6) Bauer, M.; Schroeder, S.; Tauhardt, L.; Kempe, K.; Schubert, U. S.; Fischer, D. J. Polym. Sci. Part A Polym. Chem. 2013, 51 (8), 1816.

(7) Koshkina, O.; Westmeier, D.; Lang, T.; Bantz, C.; Hahlbrock, A.; Würth, C.; Resch-Genger, U.; Braun, U.; Thiermann, R.; Weise, C.; Eravci, M.; Mohr, B.; Schlaad, H.; Stauber, R. H.; Docter, D.; Bertin, A.; Maskos, M. Macromol. Biosci. 2016, No. 16, 1287.

(8) Moreadith, R. W.; Viegas, T. X.; Bentley, M. D.; Harris, J. M.; Fang, Z.; Yoon, K.; Dizman, B.; Weimer, R.; Rae, B. P.; Li, X.; Rader, C.; Standaert, D.; Olanow, W. Eur. Polym. J. 2017, $88,524$.

(9) Eskow Jaunarajs, K. L.; Standaert, D. G.; Viegas, T. X.; Bentley, M. D.; Fang, Z.; Dizman, B.; Yoon, K.; Weimer, R.; Ravenscroft, P.; Johnston, T. H.; Hill, M. P.; Brotchie, J. M.; Moreadith, R. W. Mov. Disord. 2013, 28 (12), 2.

(10) He, Z.; Wan, X.; Schulz, A.; Bludau, H.; Dobrovolskaia, M. A.; Stern, S. T.; Montgomery, S. A.; Yuan, H.; Li, Z.; Alakhova, D.; Sokolsky, M.; Darr, D. B.; Perou, C. M.; Jordan, R.; Luxenhofer, R.; Kabanov, A. V. Biomaterials 2016, 101, 296.

(11) Luxenhofer, R.; Sahay, G.; Schulz, A.; Alakhova, D.; Bronich, T. K.; Jordan, R.; Kabanov, A. V. J. Control. Release 2011, 153 (1), 73. 
(12) Jaksch, S.; Schulz, A.; Di, Z.; Luxenhofer, R.; Jordan, R.; Papadakis, C. M. Macromol. Chem. Phys. 2016, 217 (13), 1448.

(13) Seo, Y.; Schulz, A.; Han, Y.; He, Z.; Bludau, H.; Wan, X.; Tong, J.; Bronich, T. K.; Sokolsky, M.; Luxenhofer, R.; Jordan, R.; Kabanov, A. V. Polym. Adv. Technol. 2015, 26 (7), 837.

(14) Sedlacek, O.; Monnery, B. D.; Mattova, J.; Kucka, J.; Panek, J.; Janouskova, O.; Hocherl, A.; Verbraeken, B.; Vergaelen, M.; Zadinova, M.; Hoogenboom, R.; Hruby, M. Biomaterials 2017, 146, 1.

(15) Romio, M.; Morgese, G.; Trachsel, L.; Babity, S.; Paradisi, C.; Brambilla, D.; Benetti, E. M. Biomacromolecules 2018, 19 (1), 103.

(16) Konradi, R.; Pidhatika, B.; Mühlebach, A.; Textor, M. Langmuir 2008, 24 (3), 613.

(17) Pidhatika, B.; Rodenstein, M.; Chen, Y.; Rakhmatullina, E.; Mühlebach, A.; Acikgöz, C.; Textor, M.; Konradi, R. Biointerphases 2012, 7 (1-4).

(18) Moroni, L.; Klein Gunnewiek, M.; Benetti, E. M. Acta Biomater. 2014, 10 (6), 2367.

(19) Waschinski, C. J.; Tiller, J. C. Biomacromolecules 2005, 6 (1), 235.

(20) Correia, V. G.; Ferraria, A. M.; Pinho, M. G.; Aguiar-Ricardo, A. Biomacromolecules 2015, 16 (12), 3904.

(21) Schumpert, C. A.; Dudycha, J. L.; Patel, R. C. BMC Biotechnol. 2015, 15 (1), 91.

(22) Kem, K. M. J. Polym. Sci. Polym. Chem. Ed. 1979, 17, 1977.

(23) Van Kuringen, H. P. C.; De La Rosa, V. R.; Fijten, M. W. M.; Heuts, J. P. A.; Hoogenboom, R. Macromol. Rapid Commun. 2012, 33 (9), 827.

(24) Fernandes, J. C.; Qiu, X.; Winnik, F. M.; Benderdour, M.; Zhang, X.; Dai, K.; Shi, Q. Int. J. Nanomedicine 2013, 8, 4091.

(25) Mees, M.; Haladjova, E.; Momekova, D.; Momekov, G.; Shestakova, P. S.; Tsvetanov, C. B.; Hoogenboom, R.; Rangelov, S. Biomacromolecules 2016, 17 (11), 3580.

(26) Shah, R.; Kronekova, Z.; Zahoranová, A.; Roller, L.; Saha, N.; Saha, P.; Kronek, J. J. Mater. Sci. Mater. Med. 2015, 26 (4), 157.

(27) Lungwitz, U.; Breunig, M.; Blunk, T.; Göpferich, A. Eur. J. Pharm. Biopharm. 2005, 60, 247.

(28) Höbel, S.; Aigner, A. Wiley Interdisciplinary Reviews: Nanomedicine and Nanobiotechnology. 2013, pp 484-501.

(29) Pandey, A. P.; Sawant, K. K. Mater. Sci. Eng. C 2016, 68, 904.

(30) Taranejoo, S.; Liu, J.; Verma, P.; Hourigan, K. Journal of Applied Polymer Science. 2015,.

(31) Mees, M. A.; Effenberg, C.; Appelhans, D.; Hoogenboom, R. Biomacromolecules 2016, 17 (12).

(32) Jäger, M.; Schubert, S.; Ochrimenko, S.; Fischer, D.; Schubert, U. S. Chem. Soc. Rev. 2012, $41(13), 4755$.

(33) Englert, C.; Hartlieb, M.; Bellstedt, P.; Kempe, K.; Yang, C.; Chu, S. K.; Ke, X.; Garcĺa, J. M.; Ono, R. J.; Fevre, M.; Wojtecki, R. J.; Schubert, U. S.; Yang, Y. Y.; Hedrick, J. L. Macromolecules 2015, 48 (20), 7420.

(34) Seeliger, W.; Aufderhaar, E.; Diepers, W.; Feinauer, R.; Nehring, R.; Thier, W.; Hellmann, H. Angew. Chem. Int. Ed. Engl. 1966, 5 (10), 875. 
(35) Lee, M. S.; Kim, M. G.; Jang, Y. L.; Lee, K.; Kim, T. G.; Kim, S. H.; Park, T. G.; Kim, H. T.; Jeong, J. H. Macromol. Res. 2011, 19 (7), 688.

(36) Kobayashi, S.; Hiroishi, K.; Tokunoh, M.; Saegusa, T. Macromolecules 1987, 20, 1496.

(37) Kobayashi, S. Prog. Polym. Sci. 1990, 15 (5), 751.

(38) Mannschott, C.; Hockera, H.; Kern, W. Makromoleculair Chem. 1981, 1350, 1337.

(39) Munir, A.; Goethals, E. J. Journa Polym. Sci. 1994, 19 (1981), 1985.

(40) Kern, W.; Mannschott, C.; H, H. Makromoleculair Chem. 1982, 183, 1413.

(41) Weyts, K. R.; Goethals, E. J. Polym. Bull. 1988, 19, 13.

(42) Rieger, E.; Gleede, T.; Manhart, A.; Lamla, M.; Wurm, F. R. ACS Macro Lett. 2018, 7, 598.

(43) Stewart, I. C.; Lee, C. C.; Bergman, R. G.; Toste, F. D. J. Am. Chem. Soc. 2005, 127 (50), 17616.

(44) Homann-Müller, T.; Rieger, E.; Alkan, A.; Wurm, F. R. Polym. Chem. 2016, 7 (35), 5501.

(45) Rieger, E.; Manhart, A.; Wurm, F. R. ACS Macro Lett. 2016, 5 (2), 195.

(46) Gleede, T.; Rieger, E.; Homann-Müller, T.; Wurm, F. R. Macromol. Chem. Phys. 2018, 219 (1), 1.

(47) Reisman, L.; Mbarushimana, C. P.; Cassidy, S. J.; Rupar, P. A. ACS Macro Lett. 2016, 5 (10), 1137.

(48) Herlem, G.; Lakard, B. J. Chem. Phys. 2004, 120 (19), 9376.

(49) Ziebarth, J. D.; Wang, Y. Biomacromolecules 2010, 11 (1), 29.

(50) Smits, R. G.; Koper, G. J. M.; Mandel, M. J. Phys. Chem. 1993, 97 (21), 5745.

(51) Weyts, K.; Goethals, E. Die Makromol. Chemie, Rapid Commun. 1989, 10, 299.

(52) Saegusa, T.; Fujii, H.; Ikeda, H. Macromolecules 1972, 37 (1), 108.

(53) Tanaka, R.; Ueoka, I.; Takaki, Y.; Kataoka, K.; Saito, S. Macromolecules 1983, 16 (2), 849.

(54) Monnery, B. D.; Shaunak, S.; Thanou, M.; Steinke, J. H. G. Macromolecules 2015, 48 (10), 3197.

(55) Saegusa, T.; Kobayashi, S.; Yamada, A. Macromolecules 1975, 8 (4), 390.

(56) Chujo, Y.; Sada, K.; Saegusa, T. Macromolecules 1990, 23 (10), 2693.

(57) Chujo, Y.; Yutaka, Y.; Sada, K.; Saegusa, T. Macromolecules 1989, 22 (3), 1074.

(58) Saegusa, T.; Ikeda, H.; Fujii, H. Macromolecules 1972, 5 (4), 1970.

(59) Saegusa, T.; Ikeda, H.; Fujii, H. Polymer Journal. 1972, pp 176-180.

(60) Chu, V. P.; Overberger, C. G. J. Polym. Sci. Part A-1 1986, 24, 2657.

(61) Lambermont-Thijs, H. M. L.; Heuts, J. P. A.; Hoeppener, S.; Hoogenboom, R.; Schubert, U. S. Polym. Chem. 2011, 2 (2), 313.

(62) de la Rosa, V. R.; Bauwens, E.; Monnery, B. D.; De Geest, B. G.; Hoogenboom, R. Polym. Chem. 2014, 5, 4957.

(63) Van Kuringen, H. P. C.; Lenoir, J.; Adriaens, E.; Bender, J.; De Geest, B. G.; Hoogenboom, R. Macromol. Biosci. 2012, 12 (8), 1114.

(64) Lambermont-Thijs, H. M. L.; van der Woerdt, F. S.; Baumgaertel, A.; Bonami, L.; Du Prez, F. E.; Schubert, U. S.; Hoogenboom, R. Macromolecules 2010, 43 (2), 927.

(65) Litt, M. H.; Lin, C. S. J. Polym. Sci. Part A-1 Polym. Chem. 1992, $30,779$. 
(66) Jeong, J. H.; Song, S. H.; Lim, D. W.; Lee, H.; Park, T. G. J. Control. release 2001, 73 (2-3), 391.

(67) Vlassi, E.; Pispas, S. Macromol. Chem. Phys. 2015, 216, 873.

(68) Tauhardt, L.; Kempe, K.; Schubert, U. S. J. Polym. Sci. Part A Polym. Chem. 2012, 50 (21), 4516.

(69) Jin, R. H. ChemPhysChem 2003, 4 (10), 1118.

(70) Kichler, A.; Chillon, M.; Leborgne, C.; Danos, O.; Frisch, B. J. Control. Release 2002, 81 (3), 379.

(71) Ochrimenko, S.; Vollrath, A.; Tauhardt, L.; Kempe, K.; Schubert, S.; Schubert, U. S.; Fischer, D. Carbohydr. Polym. 2014, 113, 597.

(72) Takemoto, K.; Inaki, Y. In Advances in Polymer Science; 1981.

(73) Englert, C.; Trützschler, A.-K.; Raasch, M.; Bus, T.; Borchers, P.; Mosig, A. S.; Traeger, A.; Schubert, U. S. J. Control. Release 2016, 241, 1.

(74) Hartlieb, M.; Pretzel, D.; Englert, C.; Hentschel, M.; Kempe, K.; Gottschaldt, M.; Schubert, U. S. Biomacromolecules 2014, 15 (6), 1970.

(75) Ludwick, A. G.; Overberger, C. G. J. Polym. Sci. Polym. Chem. Ed. 1982, 20, 2123.

(76) Ludwick, A. G.; Robinson, K. S.; Mccloud, J. J. Polym. Sci. Polym. Symp. 1986, 74 (1), 55.

(77) Overberger, C. G.; Kikyotani, S. J. Polym. Sci. Polym. Chem. Ed. 1983, 21 (2), 525.

(78) Wada, T.; Inaki, Y.; Takemoto, K. Polym. J. 1988, 20 (1), 1059.

(79) Lambermont-Thijs, H. M. L.; Bonami, L.; Du Prez, F. E.; Hoogenboom, R. Polym. Chem. 2010, 1 (5), 747.

(80) Saegusa, T.; Kobayashi, S.; Yamada, A. J. Appl. Polym. Sci. 1977, $21,2481$.

(81) Masson, P.; Heinrich, B.; Frère, Y.; Gramain, P. Macromol. Chem. Phys. 1994, 1212, 1199.

(82) Pavlisko, J. A.; Overberger, C. G. Journa Polym. Sci. 1981, 19, 1621.

(83) Cheng, L.; Abhilas, K. G.; Breslow, R. Proc. Natl. Acad. Sci. U. S. A. 2012, 109 (32), 12884.

(84) Sayari, A.; Heydari-Gorji, A.; Yang, Y. J. Am. Chem. Soc. 2012, 134 (33), 13834.

(85) Sayari, A.; Belmabkhout, Y. J. Am. Chem. Soc. 2010, 132 (18), 6312.

(86) Schroeder, A.; Dahlman, J. E.; Sahay, G.; Love, K. T.; Jiang, S.; Eltoukhy, A. A.; Levins, C. G.; Wang, Y.; Anderson, D. G. J. Control. Release 2012, 160 (2), 172.

(87) Kim, H.; Bae, Y. M.; Kim, H. A.; Hyun, H.; Yu, G. S.; Choi, J. S.; Lee, M. J. Cell. Biochem. 2010, 110 (3), 743.

(88) Saegusa, T.; Kobayashi, S.; Yamada, A. Polym. J. 1978, 10 (4), 403.

(89) Doyle, R. P.; Chen, X.; Macrae, M.; Srungavarapu, A.; Smith, L. J.; Gopinadhan, M.; Osuji, C. O.; Granados-Focil, S. Macromolecules 2014, 47 (10), 3401.

(90) Fukuda, Y.; Abe, D.; Tanaka, Y.; Uchida, J.; Suzuki, N.; Miyai, T.; Sasanuma, Y. Polym. J. 2016, 48 (11), 1065.

(91) Englert, C.; Fevre, M.; Wojtecki, R. J.; Cheng, W.; Xu, Q.; Yang, C.; Ke, X.; Hartlieb, M.; Kempe, K.; García, J. M.; Ono, R. J.; Schubert, U. S.; Yang, Y. Y.; Hedrick, J. L. Polym. Chem. 2016, 7 (37), 5862.

(92) Liu, X.; Yang, J. W.; Lynn, D. M. Biomacromolecules 2008, 9 (7), 2063. 
(93) Liu, X.; Yang, J. W.; Miller, A. D.; Nack, E. A.; Lynn, D. M. Macromolecules 2005, 38 (19), 7907.

(94) Bertrand, E.; Gonçalves, C.; Billiet, L.; Gomez, J. P.; Pichon, C.; Cheradame, H.; Midoux, P.; Guégan, P. Chem. Commun. (Camb). 2011, 47 (46), 12547.

(95) Verbraeken, B.; Monnery, B. D.; Lava, K.; Hoogenboom, R. Eur. Polym. J. 2017, 88, 451.

(96) Hoogenboom, R.; Monnery, B. D. Method for the preparation of uniform, High molar mass cyclic imino ether polymers. WO 2016:008817, 2016.

(97) Chujo, Y.; Sada, K.; Nomura, R.; Naka, A.; Saegusa, T. Macromolecules 1993, 26 (21), 5611.

(98) Chujo, Y.; Sada, K.; Naka, a; Nomura, R.; Saegusa, T. Macromolecules 1993, 26 (5), 883.

(99) Chujo, Y.; Sada, K.; Saegusa, T. Macromolecules 1993, 26 (24), 6320.

(100) Chujo, Y.; Sada, K.; Saegusa, T. Polym. J. 1993, 6, 599.

(101) Chujo, Y.; Sada, K.; Saegusa, T. Macromolecules 1990, 23, 2636.

(102) Guo, C.; Zhou, L.; Lv, J. Polym. Polym. Compos. 2013, 21 (7), 449.

(103) Mees, M. A.; Hoogenboom, R. Macromolecules 2015, 48 (11), 3531.

(104) Chen, C.-H.; Niko, Y.; Konishi, G. RSC Adv. 2016, 6 (49), 42962.

(105) Legros, C.; Wirotius, A.; Tam, K. C.; Taton, D. Biomacromolecules 2015, 16, 183.

(106) Legros, C.; De Pauw-Gillet, M.-C.; Tam, K. C.; Lecommmandoux, S.; Taton, D. Polym. Chem. 2013, 4 (17), 4801.

(107) Filippov, S. K.; Verbraeken, B.; Konarev, P. V.; Svergun, D. I.; Angelov, B.; Vishnevetskaya, N. S.; Papadakis, C. M.; Rogers, S.; Radulescu, A.; Courtin, T.; Martins, J. C.; Starovoytova, L.; Hruby, M.; Stepanek, P.; Kravchenko, V. S.; Potemkin, I. I.; Hoogenboom, R. J. Phys. Chem. Lett. 2017, 8 (16), 3800. 